\title{
A Camera-Aided Legibility Assessment Protocol of Displays for Enhanced Human-Computer Interaction
}

\author{
Hongyi Cai \\ Civil, Environmental \& Architectural Engineering, University of Kansas, \\ 1530 W. 15th Street, Lawrence, Kansas 66045, USA \\ hycai@ku.edu
}

\begin{abstract}
Legible text and graphics presented on computer displays and projection screens are essential for benefit of human-computer interaction. Legibility of characters depends on the display brightness, luminance contrast of characters, character size, font types, color, viewing distance and angle, and observer's acuity level. In sign and display industry, a legibility index, defined as the viewing distance divided by the character height, has been widely used for legibility evaluation. However, this index fails to examine all major factors other than geometry. To enhance human-computer interaction, a quantitative legibility evaluation method, which takes into account all major affecting factors, is needed for quick and reliable guidance, goal of this study. This study thus developed a legibility assessment protocol based on a redefined legibility index as the inverse square root of the solid angle subtended by the target, a legibility equation, and innovative camera-aided high dynamic range photogrammetric techniques the author recently developed.
\end{abstract}

Keywords: human-computer interaction, camera, high dynamic range photogrammetric techniques, legibility, equation, index.

Legibility index for perpendicular viewing

Redefined legibility index for both perpendicular and non-perpendicular viewing

Solid angle subtended by any text and graphics to the retina of the observer's eyes

Incident angle between the display normal and the sightline of an observer

Viewing distance from the observer to the display viewed at angles

Normal text height

Visual angle subtended by the height of the character

Height-to-strokewidth-ratio of text

Denominator in the Snellen ratio of the observer's acuity level

Background luminance (i.e., the luminance of displays)

World coordinates

Local coordinates of target plane

Pixel coordinate on HDR image

Camera yaw angle along $\mathrm{Z}$ axis

Camera pitch angle along $\mathrm{X}$ axis

Camera roll angle along the shooting line ( $\mathrm{Y}$ axis)

Target yaw angle along $\mathrm{Z}$ axis

Target vertical pitch angle along $X$ axis

Light source roll angle along $\mathrm{Y}$ axis

Horizontal off-axis viewing from target to camera

Vertical off-axis viewing angle from target to camera

A. Marcus (Ed.): Design, User Experience, and Usability, Pt II, HCII 2011, LNCS 6770, pp. 359-367, 2011.

(c) Springer-Verlag Berlin Heidelberg 2011 


\section{Introduction}

Legible text and graphics presented on computer displays and projection screens are essential for benefit of human-computer interaction. Legibility of characters depends on many factors, including the display brightness (i.e., the background luminance of the characters), luminance contrast of characters, character size, font types, color, viewing distance and angle, and observer's acuity level. Some empirical guidelines for legible displays are available in the literature. Table 1 summarizes these guidelines for defining an optimal cone area for locating seats with legible view of images presented on projection screens and video monitors. The horizontal fan-shaped areas were defined using a viewing angle $\varphi$, which is the angle between the display normal and the edge of the optimal view cone, and an optimum viewing distance $d$, which is in proportion to the display size (usually width is used) $[1,2,3,4,5,6,7]$. In Table 1 , note that the size of characters is not used by the guidelines for prediction, neither lighting, nor viewers' acuity, which are all essential for legibility.

Table 1. Fan-shaped ideal viewing area defined using the existing guidelines

\begin{tabular}{llll}
\hline $\begin{array}{l}\text { Display } \\
\text { types }\end{array}$ & Sources & Fan-shaped ideal viewing area (d is & $\begin{array}{l}\text { Recommendation } \\
\text { viewing distance, } \varphi \text { is horizontal } \\
\text { biewis } \\
\end{array}$ \\
& & \\
& &
\end{tabular}

\begin{tabular}{|c|c|c|c|}
\hline \multirow[t]{5}{*}{$\begin{array}{l}\text { Projection } \\
\text { screens }\end{array}$} & Aschoff [2] & $\begin{array}{l}2 \mathrm{w} \leq \mathrm{d} \leq 6 \mathrm{w}, \varphi= \pm 30^{\circ}, \text { with } \\
\text { maximum elevation angle of } 35^{\circ}, \\
\text { and depression angle of } 12^{\circ} .\end{array}$ & DIN108 \\
\hline & Hauf et al. $[1,7]$ & \multirow{2}{*}{$\begin{array}{l}2 \mathrm{w} \leq \mathrm{d} \leq 6-10 \mathrm{w}, \varphi= \pm 30^{\circ}-60^{\circ} \text {, with } \\
\text { maximum elevation angle of } 15^{\circ} \text {. } \\
1.5-2 \mathrm{w} \leq \mathrm{d} \leq 6 \mathrm{w}\end{array}$} & $\begin{array}{l}\text { No experimental } \\
\text { evidence }\end{array}$ \\
\hline & Conway [8] & & None given \\
\hline & McGowan \& Kruse [9] & \multirow{2}{*}{$\begin{array}{l}1.5 \mathrm{w} \leq \mathrm{d} \leq 4-5 \mathrm{w}, \varphi= \pm 45^{\circ} \\
1.3 \mathrm{w} \leq \mathrm{d} \leq 4.5-6 \mathrm{w}\end{array}$} & None given \\
\hline & $\begin{array}{l}\text { Online projection cal- } \\
\text { culator [10] }\end{array}$ & & $\begin{array}{l}\text { Manufacturer } \\
\text { supplied data }\end{array}$ \\
\hline \multirow{4}{*}{$\begin{array}{l}\text { Video } \\
\text { monitors }\end{array}$} & Hauf et al. [1] & $4 w \leq d \leq 12 w(14 w$ for less optimum & \multirow{2}{*}{$\begin{array}{l}\text { No experimental } \\
\text { evidence }\end{array}$} \\
\hline & & $\begin{array}{l}\text { condition), } \varphi= \pm 35^{\circ}-40^{\circ}\left( \pm 45^{\circ} \text { for }\right. \\
\text { less optimum condition), with the } \\
\text { maximum elevation angle of } 15^{\circ} \text { to } \\
\text { the bottom of image }\left(30^{\circ} \text { for less }\right. \\
\text { optimum condition). }\end{array}$ & \\
\hline & Allen et al. [4] & $\begin{array}{l}1.5 \mathrm{w}(\text { optimum } 2 \mathrm{w}) \leq \mathrm{d} \leq 6 \mathrm{w} \text { (or } 4 \mathrm{w} \\
\text { for electronic projection), } \mathrm{d}_{\max } \leq 1 \\
\mathrm{ft} / \text { in of } \mathrm{TV} \text { diagonal size; a maximum } \\
\text { elevation angle of } 35^{\circ} \text {. }\end{array}$ & None given \\
\hline & McGowan \& Kruse [9] & $2-2.5 \mathrm{w} \leq \mathrm{d} \leq 6-7 \mathrm{w}, \varphi= \pm 45^{\circ}$ & None given \\
\hline
\end{tabular}

The effect of display resolutions on the legibility of characters was also considered in the literature. The minimum acceptable displays resolution guided in 2001 was about 58 dpi [8], which is not a concern any more with today's display technologies. Sizes of characters were also considered in the literature. Human eyes can recognize 
the smallest characters subtended 1 minute of arc, yet minimum of 16, better 20 to 22 minutes of arc (or arcminute) were recommended [8]. For example, Table 2 listed the minimum character height recommended by ANSI standard for legibility [8].

Table 2. Minimum character height for legibility and the distance-to-height ratios [8]

\begin{tabular}{ccccc}
\hline $\begin{array}{c}\text { Viewing distance D } \\
\text { (feet) }\end{array}$ & \multicolumn{2}{c}{$\begin{array}{c}\text { Minimum character } \\
\text { height }\end{array}$} & \multicolumn{2}{c}{ D/H ratio (ft/in) } \\
16 & $\begin{array}{c}21 \\
\text { arcminute }\end{array}$ & $\begin{array}{c}16 \text { arcmi- } \\
\text { arcmute }\end{array}$ & $\begin{array}{c}21 \\
\text { nute }\end{array}$ & arcminute \\
\hline 5 & 0.28 & 0.37 & 17.9 & 13.5 \\
10 & 0.56 & 0.73 & 17.9 & 13.7 \\
15 & 0.84 & 1.10 & 17.9 & 13.6 \\
20 & 1.12 & 1.47 & 17.9 & 13.6 \\
25 & 1.40 & 1.83 & 17.9 & 13.7 \\
30 & 1.68 & 2.20 & 17.9 & 13.6 \\
35 & 1.96 & 2.57 & 17.9 & 13.6 \\
40 & 2.24 & 2.93 & 17.9 & 13.7 \\
\hline
\end{tabular}

To quantify all the efforts, a legibility index has been widely used in sign and display industry for evaluation of legibility levels of text and graphics. This legibility index, defined as the distance at which material can be read with perfect accuracy (the legibility distance) divided by the character height, as (1). This ratio equals the inverse tangent of the visual angle subtended by the character. Table 2 also shows $D / H$ ratios in a practical unit of ft/in, which are commonly used in sign industry. Manufacturers of computer projectors often recommended in their product manuals optimized settings for legible screens based on such $\mathrm{D} / \mathrm{H}$ ratios. However, this definition assumes that the viewing material is perpendicular to the observer, which is not always true. In practice, people often view computer displays and projection screens at different angles. Also, the conventional legibility index does not take into account other critical factors except for viewing distance and character height.

$$
L I=D / H=1 / \tan (V)
$$

To examine the legibility of characters viewed not perpendicular to the display, Cai and Green [9] redefined the legibility index as the inverse square root of the solid angle subtended by the target, as (2). This new definition was proven in laboratory experiments in that the three-dimensional solid angle, rather than the two-dimensional visual angle, both subtended by the character, captures how people recognize text and graphics that usually have two significant dimensions (width and height) [9]. In addition, Cai [10] proposed a new legibility equation, as (3) based on existing Howett's equation [11], which was later proven in two laboratory experiments using human subjects [10]. Equation (3) predicts legibility of text presented on assumed matte displays surfaces without glare sources visible at the peripheral of the field of view, and recognized by young observers at threshold (just readable) 100\% accuracy for incident angles from $0^{\circ}$ to $82.8^{\circ}$, which include nearly all possible viewing angles in human-computer interactions. Equation (3) has taken into account nearly all the major 
factors of legibility, including incident angle, height, distance, height-to-strokewidth ratio, Snellen visual acuity, background luminance, and luminance contrast.

$$
\begin{gathered}
L I^{\prime}=\sqrt{1 / \omega} \\
H= \begin{cases}4.1 \times 10^{-4} \cdot \frac{H}{S_{w}} \cdot D \cdot S_{d} \cdot L_{b}^{-0.213} \cdot C_{\%}{ }^{-0.532} \cdot(\cos \xi)^{-0.5} & 0^{\circ} \leq \xi \leq 65.7^{\circ} \\
4.1 \times 10^{-4} \cdot \frac{H}{S_{w}} \cdot D \cdot S_{d} \cdot L_{b}^{-0.213} \cdot C_{\%}^{-0.532} \cdot(\cos \xi)^{-0.5} \cdot(0.024 \xi-0.577) & 65.7^{\circ}<\xi \leq 82.8^{\circ}\end{cases}
\end{gathered}
$$

\section{Research Problems}

To enhance human-computer interaction, a quantitative legibility evaluation method is needed for quick and reliable guidance, goal of this study. Such evaluation method will use equations (2) and (3) that the author has developed. However, for calculating legibility of displays, inputs to those two equations would be hindered by the conventional light and geometry measurement techniques using light meters, rules, etc.

The conventional way of light measurement by using meters, such as Minolta illuminance meter T-10 and luminance meter LS-100, has not dramatically changed in the past 70 years, since the first light meter, probably the AVO Smethurst High-Light exposure meter for photography, was made in 1937 [12], although meters have been continuously improved for enhanced precision and reduced size. Conventionally, light meters are either held on hands or mounted on tripod to measure often non-uniform light distributed on light-emitting and non-luminous surfaces. This is a point-by-point process, very tedious, basically incapable to measure large areas or the entire scene. In addition, those meters read an average value of illuminance or luminance at every measuring point, by simply treating non-uniform light fallen on photosensors of meters as uniform. These limitations have negative effect on lighting profession and hamper its development. Measuring common non-uniform light is thus beyond the capability of conventional meters. Basically, meters are incapable for common dynamic human-computer interaction where viewing angles and interior lighting conditions keep changing.

This situation was changed recently (2000s). Emerging high dynamic range (HDR) photography techniques could acquire luminance distribution of an entire static scene within 1-2 minutes, which is the camera shooting time, in extremely high measuring resolution [13]. Such innovative techniques use inexpensive consumer grade digital cameras, often fitted with wide-angle lens (e.g., fisheye and zoom lens), as the test rig. First, a series of low dynamic range (LDR) photographs are taken with sequential exposures, which are then fused into an HDR image by using some data-fusion software, such as Photosphere, hdrgen, Radiance, and Photolux [13]. The HDR image encloses all luminance data of the entire test scenario at pixel level. Depending on the pixel resolution of the camera sensor (e.g., 7,962,624 pixels for Canon EOS Rebel XT, 17,915,904 pixels for Canon EOS Rebel T2i), each HDR image may have acquired millions or billions of luminance values of the test scenario. Therefore, the HDR photography techniques have great potentials to overcome those limitations of conventional light measurement using meters. The HDR photography techniques have 
been proven reliable with acceptable accuracy in a recent study carried out by Cai and Chung [13] and Inanici [14].

Unfortunately, two inherent problems of the HDR photography techniques have hindered their applications for light measurement [15]. First, most wide-angle lenses, in particular fisheye lenses, have radical lens distortion, as shown in Fig. 1, including barrel and pin-cushion distortions. Distorted geometries of luminous elements, which cannot be corrected by using the HDR photography techniques solely, thus encumber the application of HDR images for legibility assessment. Second, except for luminance, HDR photography techniques cannot measure geometries of luminous elements required for lighting quality and legibility assessment. The coordinates in real world of any pixel on the HDR images cannot be identified by HDR photography techniques.
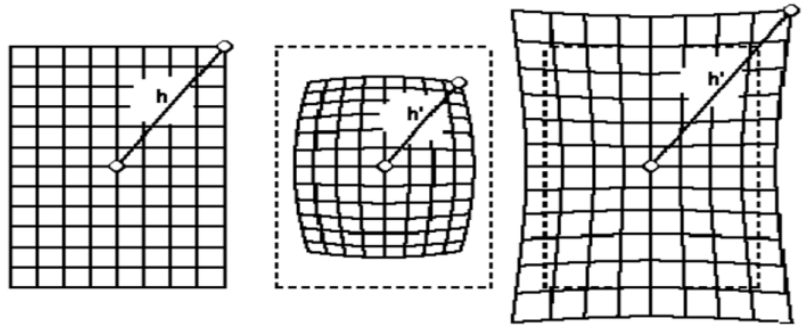

Fig. 1. Radical lens distortion [16]

\section{HDR Photogrammetric Techniques}

To solve this problem, this study employed an innovative camera-aided measurement method - high dynamic range (HDR) photogrammetric techniques recently developed by the author [15]. Such HDR photogrammetric techniques integrate long established photogrammetric techniques for geometric measurement into the emerging HDR photography techniques to measure both luminance and geometries of the entire scene at pixel level in a quick, reliable, and holistic way. Applied in human-computer interaction, the HDR photogrammetric techniques can measure both light and geometries simultaneously across the entire human-computer interfaces in 1-2 minutes. An HDR image fused using HDR photogrammetric techniques encloses all luminance and geometric value across the entire scene at pixel level. Such innovative HDR photogrammetric techniques will surely stimulate applications in human-computer interactions. The HDR photogrammetric coordinates and equations are introduced below.

\subsection{HDR Photogrammetric Coordinates}

The collinearity relationship between image and object coordinates used to develop the HDR photogrammetric techniques are illustrated in Fig. 2 [15]. As shown in Fig. 2 , a consumer grade digital camera is mounted at the focal point $\mathrm{O}(0,0,0)$ (assumed at zero point $(0,0,0)$, could be at any point $\mathrm{O}\left(\mathrm{X}_{\mathrm{o}}, \mathrm{Y}_{\mathrm{o}}, \mathrm{Z}_{\mathrm{o}}\right)$ ), with yaw angle $\kappa$, pitch angle $\eta$, and roll angle $\varphi$ related to the world coordinates XYZ, following the right 
hand rule. The HDR image plane, located on the image sensor of the camera, has two dimensional pixel coordinates $\mathrm{xz}$. The principal point is located at $\mathrm{c}\left(\mathrm{x}_{\mathrm{c}}, \mathrm{z}_{\mathrm{c}}\right)$. The target plane is the one in blue color, with local coordinates $X^{\prime} Y^{\prime} Z^{\prime}$, which also has yaw angle $\theta$, pitch angle $\tau$, and roll angle $\rho$ in light of the world coordinates XYZ, also following the right hand rule. The target $\mathrm{P}(\mathrm{X}, \mathrm{Y}, \mathrm{Z})$ and reference point $\mathrm{P}_{\mathrm{i}}\left(\mathrm{X}_{\mathrm{i}}, \mathrm{Y}_{\mathrm{i}}, \mathrm{Z}_{\mathrm{i}}\right)$ are both located on the target plane. The position of reference point $\mathrm{P}_{\mathrm{i}}\left(\mathrm{X}_{\mathrm{i}}, \mathrm{Y}_{\mathrm{i}}, \mathrm{Z}_{\mathrm{i}}\right)$ in world coordinates is measured in the field. Minimum three, ideally four reference points are needed for each target plane. Based on the photogrammetric coordinates, the location of target $\mathrm{P}(\mathrm{X}, \mathrm{Y}, \mathrm{Z})$ in world coordinates can be calculated from its pixel location $\mathrm{p}(\mathrm{x}, \mathrm{z})$ on the HDR image, aided by the reference point $\mathrm{P}_{\mathrm{i}}\left(\mathrm{X}_{\mathrm{i}}, \mathrm{Y}_{\mathrm{i}}, \mathrm{Z}_{\mathrm{i}}\right)$, by using some photogrammetric equations (4) - (12), to be introduced next.

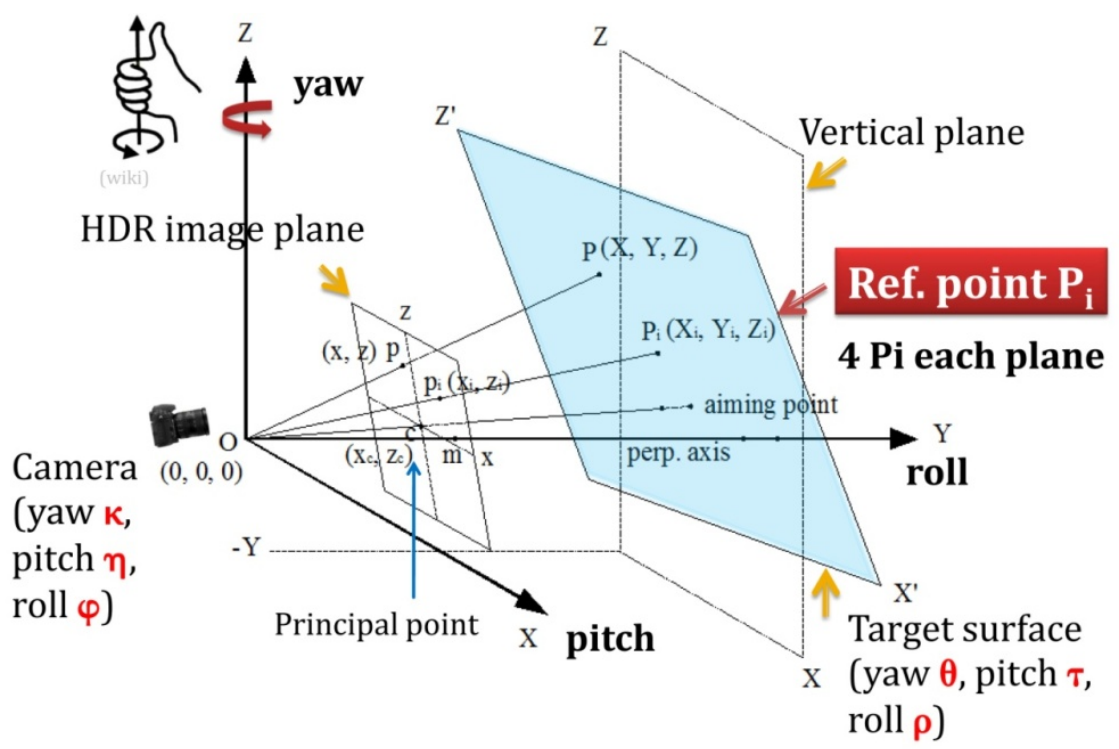

Fig. 2. HDR photogrammetric coordinates [15]

\subsection{HDR Photogrammetric Equations}

Step 1: find target point $P\left(X^{\prime}, Y^{\prime}, Z^{\prime}\right)$ in local coordinates $X^{\prime} Y^{\prime} Z^{\prime}$ on the target plane

Convert target point $\mathrm{P}(\mathrm{X}, \mathrm{Y}, \mathrm{Z})$ in world coordinates to $\mathrm{P}\left(\mathrm{X}^{\prime}, \mathrm{Y}^{\prime}, \mathrm{Z}^{\prime}\right)$ in local coordinates on the target plane [17].

$$
\left(\begin{array}{l}
X^{\prime} \\
Y^{\prime} \\
Z^{\prime}
\end{array}\right)=\left(\begin{array}{lll}
r_{11}^{\prime} & r_{12}^{\prime} & r_{13}^{\prime} \\
r_{21}^{\prime} & r_{22}^{\prime} & r_{23}^{\prime} \\
r_{31}^{\prime} & r_{32}^{\prime} & r_{33}^{\prime}
\end{array}\right)^{-1}\left(\begin{array}{l}
X \\
Y \\
Z
\end{array}\right)
$$




$$
\left(\begin{array}{lll}
r_{11}^{\prime} & r_{12}^{\prime} & r_{13}^{\prime} \\
r_{21}^{\prime} & r_{22}^{\prime} & r_{23}^{\prime} \\
r_{31}^{\prime} & r_{32}^{\prime} & r_{33}^{\prime}
\end{array}\right)=\left(\begin{array}{ccc}
\cos \rho \cos \theta & -\cos \rho \sin \theta & \sin \rho \\
\cos \tau \sin \theta+\sin \tau \sin \rho \cos \theta & \cos \tau \cos \theta-\sin \tau \sin \rho \sin \theta & -\sin \tau \cos \rho \\
\sin \tau \sin \theta-\cos \tau \sin \rho \cos \theta & \sin \tau \cos \theta+\cos \tau \sin \rho \sin \theta & \cos \tau \cos \rho
\end{array}\right)
$$

Since for orthogonal matrix $\mathrm{Q}$, its transpose is equal to its inverse, $\mathrm{Q}^{-1}=\mathrm{Q}^{\mathrm{T}}$, which applies in these situations [17].

$$
\begin{gathered}
\left(\begin{array}{lll}
r_{11}^{\prime} & r_{12}^{\prime} & r_{13}^{\prime} \\
r_{21}^{\prime} & r_{22}^{\prime} & r_{23}^{\prime} \\
r_{31}^{\prime} & r_{32}^{\prime} & r_{33}^{\prime}
\end{array}\right)^{-1}=\left(\begin{array}{cccc}
r_{11}^{\prime} & r_{12}^{\prime} & r_{13}^{\prime} \\
r_{21}^{\prime} & r_{22}^{\prime} & r_{23}^{\prime} \\
r_{31}^{\prime} & r_{32}^{\prime} & r_{33}^{\prime}
\end{array}\right)^{\mathrm{T}} \\
=\left(\begin{array}{ccc}
\cos \rho \cos \theta & \cos \tau \sin \theta+\sin \tau \sin \rho \cos \theta & \sin \tau \sin \theta-\cos \tau \sin \rho \cos \theta \\
-\cos \rho \sin \theta & \cos \tau \cos \theta-\sin \tau \sin \rho \sin \theta & \sin \tau \cos \theta+\cos \tau \sin \rho \sin \theta \\
\sin \rho & -\sin \tau \cos \rho & \cos \tau \cos \rho
\end{array}\right)
\end{gathered}
$$

For reference point $\mathrm{P}_{\mathrm{i}}\left(\mathrm{X}_{\mathrm{i}}, \mathrm{Y}_{\mathrm{i}}, \mathrm{Z}_{\mathrm{i}}\right)$, located on the target plane with yaw angle $\theta$, pitch angle $\tau$, and roll angle $\rho$, its coordinates transformation from current XYZ coordinates to $\mathrm{X}^{\prime} \mathrm{Y}^{\prime} \mathrm{Z}^{\prime}$ coordinates is below

$$
\left(\begin{array}{c}
X_{i}^{\prime} \\
Y_{i}^{\prime} \\
Z_{i}^{\prime}
\end{array}\right)=\left(\begin{array}{lll}
r_{11}^{\prime} & r_{12}^{\prime} & r_{13}^{\prime} \\
r_{21}^{\prime} & r_{22}^{\prime} & r_{23}^{\prime} \\
r_{31}^{\prime} & r_{32}^{\prime} & r_{33}^{\prime}
\end{array}\right)^{\mathrm{T}}\left(\begin{array}{c}
X_{i} \\
Y_{i} \\
Z_{i}
\end{array}\right)
$$

Likewise, the camera position $\mathrm{O}\left(\mathrm{X}_{0}, \mathrm{Y}_{0}, \mathrm{Z}_{0}\right)$ in real world coordinates $\mathrm{XYZ}$ can be transformed to $X^{\prime} Y^{\prime} Z^{\prime}$ coordinates as below. If $\mathrm{O}\left(\mathrm{X}_{0}, \mathrm{Y}_{0}, \mathrm{Z}_{0}\right)=(0,0,0)$, new coordinates will remain at $\mathrm{O}\left(\mathrm{X}_{0}^{\prime}, \mathrm{Y}_{0}^{\prime}, \mathrm{Z}_{0}^{\prime}\right)=(0,0,0)$

$$
\left(\begin{array}{c}
X_{0}^{\prime} \\
Y_{0}^{\prime} \\
Z_{0}^{\prime}
\end{array}\right)=\left(\begin{array}{lll}
r_{11}^{\prime} & r_{12}^{\prime} & r_{13}^{\prime} \\
r_{21}^{\prime} & r_{22}^{\prime} & r_{23}^{\prime} \\
r_{31}^{\prime} & r_{32}^{\prime} & r_{33}^{\prime}
\end{array}\right)^{\mathrm{T}}\left(\begin{array}{c}
X_{0} \\
Y_{0} \\
z_{0}
\end{array}\right)
$$

Therefore, target point $\mathrm{P}\left(\mathrm{X}^{\prime}, \mathrm{Y}^{\prime}, \mathrm{Z}^{\prime}\right)$ in local $\mathrm{X}^{\prime} \mathrm{Y}^{\prime} \mathrm{Z}^{\prime}$ coordinates on the target plane is calculated as below

$$
\begin{gathered}
X^{\prime}=X_{0}{ }^{\prime}+\left(Y^{\prime}-Y_{0}{ }^{\prime}\right) \frac{R_{11}\left(x-x_{c}\right)+R_{12} f+R_{13}\left(z-z_{c}\right)}{R_{21}\left(x-x_{c}\right)+R_{22} f+R_{23}\left(z-z_{c}\right)} \\
Z^{\prime}=Z_{0}{ }^{\prime}+\left(Y^{\prime}-Y_{0}{ }^{\prime}\right) \frac{R_{31}\left(x-x_{c}\right)+R_{32} f+R_{33}\left(z-z_{c}\right)}{R_{21}\left(x-x_{c}\right)+R_{22} f+R_{23}\left(z-z_{c}\right)} \\
Y^{\prime}=Y_{i}^{\prime}
\end{gathered}
$$

obtained from reference point $\mathrm{P}_{\mathrm{i}}\left(\mathrm{X}_{\mathrm{i}}^{\prime}, \mathrm{Y}_{\mathrm{i}}^{\prime}, \mathrm{Z}_{\mathrm{i}}^{\prime}\right)$, expressed in $\mathrm{X}^{\prime} \mathrm{Y}^{\prime} \mathrm{Z}^{\prime}$ coordinates, where reference point is located on the target plane 


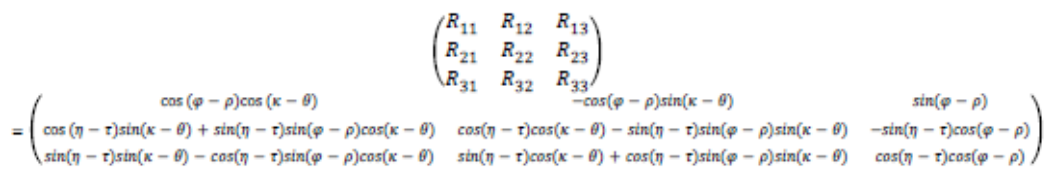

Step 2: Convert $\mathrm{P}\left(\mathrm{X}^{\prime}, \mathrm{Y}^{\prime}, \mathrm{Z}^{\prime}\right)$ in local coordinates $\mathrm{X}^{\prime} \mathrm{Y}^{\prime} \mathrm{Z}^{\prime}$ on the target plane to $\mathrm{P}(\mathrm{X}$, $\mathrm{Y}, \mathrm{Z})$ in world coordinates

Finally, convert the calculated target $\mathrm{P}\left(\mathrm{X}^{\prime}, \mathrm{Y}^{\prime}, \mathrm{Z}^{\prime}\right)$ in $\mathrm{X}^{\prime} \mathrm{Y}^{\prime} \mathrm{Z}^{\prime}$ coordinates back to $\mathrm{P}(\mathrm{X}$, $\mathrm{Y}, \mathrm{Z}$ ) in $\mathrm{XYZ}$ coordinates

$$
\begin{aligned}
&\left(\begin{array}{l}
X \\
Y \\
Z
\end{array}\right)=\left(\begin{array}{lll}
r_{11}^{\prime} & r_{12}^{\prime} & r_{13}^{\prime} \\
r_{21}^{\prime} & r_{22}^{\prime} & r_{23}^{\prime} \\
r_{31}^{\prime} & r_{32}^{\prime} & r_{33}^{\prime}
\end{array}\right)\left(\begin{array}{l}
X^{\prime} \\
Y^{\prime} \\
Z^{\prime}
\end{array}\right) \\
&\left(\begin{array}{lll}
r_{11}^{\prime} & r_{12}^{\prime} & r_{13}^{\prime} \\
r_{21}^{\prime} & r_{22}^{\prime} & r_{23}^{\prime} \\
r_{31}^{\prime} & r_{32}^{\prime} & r_{33}^{\prime}
\end{array}\right)=\left(\begin{array}{ccc}
\cos \rho \cos \theta & -\cos \rho \sin \theta & \sin \rho \\
\cos \tau \sin \theta+\sin \tau \sin \rho \cos \theta & \cos \tau \cos \theta-\sin \tau \sin \rho \sin \theta & -\sin \tau \cos \rho \\
\sin \tau \sin \theta-\cos \tau \sin \rho \cos \theta & \sin \tau \cos \theta+\cos \tau \sin \rho \sin \theta & \cos \tau \cos \rho
\end{array}\right)
\end{aligned}
$$

Using above photogrammetric equations, also aided by the reference points $P_{i}\left(X_{i}\right.$, $\left.\mathrm{Y}_{\mathrm{i}}, \mathrm{Z}_{\mathrm{i}}\right)$ measured in the field, the real world position of target $\mathrm{P}(\mathrm{X}, \mathrm{Y}, \mathrm{Z})$ in the test scene can be calculated from its pixel location $\mathrm{p}(\mathrm{x}, \mathrm{z})$ on the HDR image. At the same time, the luminance value $\mathrm{L}$ of pixel $\mathrm{p}(\mathrm{x}, \mathrm{z})$ can also be obtained from the HDR image. Therefore, with known luminance and geometry, legibility of characters presented on the displays can be evaluated at pixel level.

\section{Conclusions}

A quick and precise legibility assessment protocol of displays aided by the two legibility equations and the HDR photogrammetric techniques is thus proposed in this study for enhanced human-computer interaction, by using a consumer grade digital camera to take some photographs of the displays. HDR images fused with the HDR photogrammetric techniques can provide both luminance and world coordinates of any pixel on the screens, for legibility assessment of displays. As expected, such innovative HDR photogrammetric techniques will have a great chance to supplement or even replace the conventional legibility assessment method using meters and rules, etc. Aided by this protocol, it is also possible to develop a quick method for postoccupancy evaluation of legible human-computer interfaces. Yet it is worthy of mentioning that further studies are necessary to improve the legibility equation (3) for specular surfaces that most displays actually have.

\section{References}

1. Hauf, H.D., Koppes, W.F., Green, A.C., Gassman, M.C.: New Space for LearningDesigning College Facilities to Utilize Instructional Aids and Media. Report of a Research Project Conducted By the School Of Architecture. Rensselaer Polytechnic Institute, Troy, NY (1961) 
2. Aschoff, V.: Planning the large lecture theatre. In: Duncan, C.J. (ed.) Modern Lecture Theatres, pp. 17-25. Oriel Academic, London (1966)

3. Allen, R.L., Ault, D.K., Bowen, J.T., Clabaugh, S., Cuttill, W.J., Dewitt, B.B., et al.: Design of General-Purpose Classrooms and Lecture Halls. Pennsylvania State University, University Park (1991)

4. Hauf, H.D., Koppes, W.F., Green, A.C., Gassman, M.C., Haviland, D.S.: Revised New Spaces for Learning: the Design of College Facilities to Utilize Instructional Aids and Media. Rensselaer Polytechnic Institute, Troy (1966)

5. Conway, K.: Master Classrooms: Classroom Design with Technology in Mind. University of North Carolina at Chapel Hill, Academic Computing Publications, Inc. (1990)

6. McGowan, M., Kruse, K., (ed.): Interior Graphic Standards. John Wiley \& Sons, Hoboken (2003)

7. Projector central (n.d.). Projection Calculator Pro, http: / /www.projectorcentral.com/projection-calculatorpro. Cfm (retrieved October 30, 2009)

8. Musgrave, G.: Legibility of projected information (2001), http: / /www. conceptron.com/articles/pdf/legibility_of_project ed_information.pdf (retrieved January 15, 2011)

9. Cai, H., Green, P.A.: Legibility Index to Examine Common Viewing Situations: A New Definition Using Solid Angle. LEUKOS: The Journal of the Illuminating Engineering Society of North America 5(4), 279-295 (2009)

10. Cai, H.: Legibility Equation for Determining Ideal Viewing Areas in Lecture Halls (dissertation). University of Michigan, Ann Arbor (2008); available from ProQuest Information and Learning, Ann Arbor, MI; AAT 3304934

11. Howett, G.L.: Size of Letters Required for Visibility As a Function of ViewingDistance and Observer Visual Acuity. National bureau of standards, Washington D.C. (1983)

12. Ollinger, J. Smethurst High-Light exposure meter, http://www.jollinger.com/ photo/meters/meters/avo_smethurst.html (retrieved December 24, 2010)

13. Cai, H., Chung, T.M.: Improving the Quality of High Dynamic Range Images. Lighting Research \& Technology, OnlineFirst (2010)

14. Inanici, M.N.: Evaluation of high dynamic range photography as a luminance data acquisition system. Lighting Research \& Technology 38(2), 123-136 (2006)

15. Cai, H., Chung, T.M.: Introduction to high dynamic range photogrammetric techniques: beginning of a new lighting stage. In: IES Annual Conference 2010, Toronto, Canada, November 7-9 (2010)

16. Das, A., Patil, D.: Efficient Measurement and Correction techniques for Lens Distortion. EE362 Final project (2006), http://scien.stanford.edu/pages/labsite/ 2006 /psych221/projects / 06 /ddpatil / (retrieved on May 14, 2010)

17. Kraus, K.: Photogrammetry - Geometry from Images And Laser Scans, 2nd edn. Walter De Gruyter, Berlin (2007) 